\title{
Molecular dynamics study of liquid silica under high pressure
}

\author{
Akira Takada ${ }^{1,3}$, Robert G. Bell ${ }^{2}$, C. Richard A. Catlow ${ }^{2}$ \\ ${ }^{1}$ Research Center, Asahi Glass Co. Ltd., 1150 Hazawa-cho, Yokohama 221-8755, Japan \\ ${ }^{2}$ Dept of Chemistry, University College London, 20 Gordon St., London WC1H OAJ, UK \\ ${ }^{3}$ Dept of Earth Sciences, University College London, Gower St., London W1E 6BT, UK
}

\begin{abstract}
Structural changes of liquid silica are investigated under high pressure by molecular dynamics simulation. It is well known that high-silica liquids display anomalous pressure-dependent behaviour in their diffusivities. The potential model, the so-called 'soft potential', is used, as it is expected to simulate the structural changes of silica at high temperature well. With increasing pressure, above the glass transition temperature, the simulated silica melt shows the so-called diffusivity maximum under a pressure of $20 \mathrm{GPa}$, as already shown by the previous studies. However, it is also found that this diffusivity maximum disappears above $2800 \mathrm{~K}$. The analysis of Si coordination number suggests that the competition between the increase of five-fold and that of three-fold controls the extent of the anomaly. Secondly, the analysis of 'local oxygen packing number (LOPN)', that had been developed to investigate geometrical features in amorphous structures, is applied. In a complementary manner to the analysis of the Si coordination number, the local structure in the silica melt shows the gradual structural transformation from a low-density to high density packing on compression. Finally, a model explaining the two types of change of diffusivity in silica melt was proposed in combination with the LOPN analysis and the structon analysis that had been developed to investigate the thermal change of local structures.
\end{abstract}

*Corresponding author: Akira Takada

E-mail address: akira-takada@agc.com

Tel: +81-45-374-7304; Fax: +81-45-374-8893

Keywords: silica; molecular dynamics; structure; diffusion 


\section{Introduction}

It has been suggested that liquid silica shows several anomalous features under compression, such as the existence of a density maximum [1,2], a diffusivity maximum [3-6], and a specific heat anomaly [7]. It is speculated that the local density maximum appears at around $1820 \mathrm{~K}$ with an increase in temperature at ambient pressure. Several molecular dynamics (MD) simulations demonstrated the existence of the density maximum [8-11]. MD studies [8-11] also provided insight into its microscopic origin according to which the network structure of silica becomes a compact structure at the corresponding temperature. In a further detailed analysis [23,24], the 'structon analysis' suggested that the expanded structural fragments in common with the ideal $\beta$-cristobalite started corrupting and disappearing around the density maximum point. It is interesting to note that this anomaly is thought to be linked with the so-called thermo-mechanical anomaly, e.g. stiffening upon heating and softening upon cooling in silica glass. A further MD simulation explained that the increase of structure fragment similar to that observed in $\beta$-cristobalite would be the origin of such thermo-mechanical anomaly upon heating [13]. We speculate that if more $\beta$-cristobalite-like fragments exist in the glass, then more corruption of such fragments occurs leading to the system showing a stronger density maximum in the melt.

As for the diffusivity maximum, the local maximum appeared at a pressure of between 10-30 $\mathrm{GPa}$ with an increase in pressure above the glass transition temperature. After a pioneering MD study [3] using the WAC potential suggested the existence of the diffusivity maximum at $6000 \mathrm{~K}$, its subsequent study [4] correlated this with a prevalence of five-fold coordination of Si atoms. Related to the coordination change, it was proposed that the diffusion of oxygen atoms occurs through a transient structure with higher coordination [5]. In addition, another MD study [6] also indicated the existence of the diffusivity maximum at $2100 \mathrm{~K}$ under a pressure of $20 \mathrm{GPa}$ using a different potential model. The study suggested that the generation of many five-fold coordinated $\mathrm{Si}$ atoms increases the diffusivity, while, in contrast, the generation of many six-fold coordinated $\mathrm{Si}$ atoms decreases the diffusivity upon further compression. On the other hand, there is no experimental data on the existence of the diffusivity maximum for liquid silica, but several experimental data for the other silicate melt systems, e.g. albite $\left(\mathrm{NaAlSi}_{3} \mathrm{O}_{8}\right)$ and an equimolar albite/sodium tetrasilicate $\left(\mathrm{Na}_{2} \mathrm{Si}_{4} \mathrm{O}_{9}\right)$ mixture showed such tendency [14].

Related to the diffusion maximum, it is interesting to investigate how the glass composition changes the pressure dependence of the viscosity of the silicate melts around ambient pressure. The viscosity and the diffusivity are expected to have an inverse relation through the Stokes-Einstein equation. It is well known that lower-silica, highly depolymerized aluminosilicate melts behave like normal ionic liquids under compression. Their viscosity, however, increases upon compression. In 
contrast, higher-silicate melts show an anomalous behavior under compression. Their viscosity decreases upon compression. The positive and negative pressure dependence of the viscosity are explained by a decrease and increase in the entropy of mixing of bridging and non-bridging oxygen atoms [15].

As to the specific heat anomaly, the earlier MD study [7] showed that there is a heat capacity maximum around $4000 \mathrm{~K}$, at which temperature the so-called fragile-to-strong transition was also found using the same BKS potential model [16]. Therefore, this study speculated that the heat capacity anomaly is related to poly-amorphic behavior between a low-density and high-density glass. If any poly-amorphic behaviour exists under ambient pressure rather than high pressure, particular attention should be given to the point that two glasses with different density only comprise four-fold coordinated Si atoms. A more recent MD study [17] estimated that the poly-amorphic critical point occurs near $4000 \mathrm{~K}$ and $5 \mathrm{GPa}$ by using the WAC potential. In addition, the diffusivity increased monotonically over a temperature range of $3000 \mathrm{~K}$ to $8000 \mathrm{~K}$ up to a pressure of $16 \mathrm{GPa}$ [18]. For a review of progress on silica simulations over forty years focussing on the glass transition, critical points, and liquid-liquid transitions, we refer to [19].

In this study, MD simulations are used to study the structural transformations in liquid silica under high pressure. The so-called 'soft potential' [12] is used, because the potential could reproduce several high-temperature structural transformations of silica at reasonable temperatures when compared with experiment. The diffusivity of liquid silica is calculated at varying temperature and pressure. Next, the distribution of coordination number around Si atoms and the 'local oxygen packing number (LOPN)' [20] are analyzed at each (P, T) point. Finally, we discuss the dominant structural mechanism relevant to the diffusivity maximum occurring under high pressure. 


\section{Computational model}

\subsection{Potential model and molecular dynamic simulation}

Three main MD methods are now available. The most accurate is the first-principles method based on quantum mechanics [e.g. 21]. No interatomic potential model is necessary; however, calculations of diffusivity always require huge computation time. The second model employs the polarizable interatomic potential model [e.g. 22]. By taking the polarizability of the oxygen atoms with an additional degree of freedom, the model can reproduce a spectroscopic feature more precisely. However, the model still requires more computation time than the third model, i.e. the rigid ion model, especially when structure is calculated at high temperature because of slow convergence of the polarizable effects. Although this model is the most efficient in terms of computation time, the choice of interatomic potential model is very important. In this study, the rigid ion model was used enabling long time scale simulations.

Many potential models have been developed for $\mathrm{SiO}_{2}$. Each has its merits and demerits. To simulate liquid silica, it should be noted that the real boiling point of silica is around $2505 \mathrm{~K}$, suggesting that the superheated liquid state above the boiling point is in fact being used to simulate liquid silica; and the further the simulation deviates from the real experimental conditions the more difficult become real quantitative discussions. Two potential models, the 'BKS' potential [16] and the 'soft potential' model, which can be easily implemented in any MD code, were critically examined and their features compared from an objective point of view [27]. The main difference between the two models lies in the magnitude of atomic charges. The charge on the oxygen atoms is -1.2 for the BKS and -0.65 for the 'soft' model. It is noted that the magnitude of the charge is the smallest for the 'soft potential' model compared to the WAC [3], the BKS, TTAM [5] and CHIK [6] models. It is known that the BKS potential overestimates the melting temperature of crystals by more than 1000K [30]. It estimated that BKS simulations give the melting temperature of cristobalite at $3600 \mathrm{~K}$, although the experimental temperature is $2000 \mathrm{~K}$. In contrast, the 'soft' potential estimates it at $2400 \mathrm{~K}$. In addition, the 'soft potential' model can estimate the glass temperature at $1400 \mathrm{~K}$ compared with the experimental value of $1470 \mathrm{~K}$ [12]. The model has reproduced reasonably the structural changes in silica especially at high temperature $[23,24]$ due to the smaller values of the atomic charges. Therefore, the 'soft potential' model was employed in this study.

The functional forms and parameters are listed in a previous paper [12]. The number of atoms in the simulation box is 990 (330 Si and $660 \mathrm{O}$ ) in order to ensure consistency between this and a series of previous studies $[12,23,24]$. Any error analysis due to sample dependency was omitted in this study, such an analysis having been performed in the previous study [24].

The initial structures at each temperature (from $2000 \mathrm{~K}$ to $4000 \mathrm{~K}$ at $200 \mathrm{~K}$ intervals) used the 
stabilized models calculated in our previous study [12].

At each temperature, the structure was compressed stepwise from $0 \mathrm{GPa}$ up to $32 \mathrm{GPa}$ at $4 \mathrm{GPa}$ intervals and stabilized for 10ns in the NPT ensemble. After stabilization, the data calculated for 100 ns at each pressure were analyzed to calculate the diffusivity, the coordination numbers and to perform the local oxygen packing number (LOPN) and the atomistic energy distribution (AED) analysis. The diffusivity was calculated from a slope of mean square displacement curve of oxygen atoms. The calculated conditions for the coordination numbers, the LOPN, and the AED were explained in the previous studies [12,20-22]. Calculations were performed with the CONDOR High End facilities at UCL.

\section{2 'Local oxygen packing number (LOPN)' analysis}

The LOPN analysis is a geometrical modeling technique developed by the author [20], especially to investigate the local structural changes in the amorphous and liquid states on compression. Conventional modelling regards the whole structure as comprised of polyhedra that are calculated from the coordination number around a cation atom. In the case of silica system, this modeling is convenient to distinguish between polyhedra with four-fold, five-fold, and six-fold $\mathrm{Si}$ atoms. On the other hand, it cannot distinguish between the low-density and the high-density tetrahedral structures, because both are comprised of the same four-fold $\mathrm{Si}$ atoms. In contrast, the LOPN can distinguish between the low-density structure with the four-fold coordination, the high-density with four-fold, and the ultra-high-density structure with the six-fold as shown in the previous study [20]. The LOPN is calculated for each $\mathrm{O}$ atom as a number of its surrounding $\mathrm{O}$ atoms within a cut-off distance of $3.03 \AA$. In the case of silica crystal structures, the LOPN of cristobalite (or quartz), coesite and stishovite have each a sharp peak of 6 and 7, and 12. In contrast, silica glass has a broader distribution of the LOPN. It is expected that the LOPN of 5-6, 7-8, 11-12 each corresponds with the low-density with four-fold coordination, the high-density with four-fold coordination and the ultra-high-density local structure with six-fold coordination in either amorphous or liquid.

\section{3 'Structon' analysis}

The 'structon' analysis was developed to investigate the thermal structural changes in silica glass and liquids [12]. Each $\mathrm{Si}_{2} \mathrm{O}_{7}$ unit found in simulated structures was categorized into four 'structons' based on two indices that seemed to represent the structural features. As temperature increases, each structon converts from $\alpha$ - to $\delta$-structon through $\beta$ - and $\gamma$-structon. The first index is a standard deviation of torsion angles between two neighbouring $\mathrm{SiO}_{4}$ units. The index distinguishes between $\alpha-$ and $\beta$-structon. The second index is a 'bonding state' depending on temporal changes in Si-O bonds. The index distinguishes between $\beta$ - and $\gamma$ - structon, and between $\gamma-$ and $\delta$-structon. The calculated procedures are explained in Ref.26. At the moment, this analysis is only applicable to 
structures at ambient pressure and it is based on the structural unit of the $\mathrm{SiO}_{4}$ tetrahedron. In other words, the structon analysis is inapplicable to local structures with 3-, 5-, or 6-coordinated Si. In this study, the 'structon' analysis was only applied to $\mathrm{Si}_{2} \mathrm{O}_{7}$ units comprised of 4-fold coordinated $\mathrm{Si}$ atoms. All the other coordinated Si atoms rather than 4-fold coordination were discarded beforehand in this analysis. 


\section{Results}

\subsection{Diffusivity and coordination number}

The calculated diffusion constant of oxygen atoms is shown in Fig.2. All the experimental values were measured only below $1673 \mathrm{~K}$ and at ambient pressure [31,32]. The calculated value of diffusion constant of oxygen atoms at $1600 \mathrm{~K}$ is $2 \times 10^{-13} \mathrm{~cm}^{2} / \mathrm{s}$ and it lies between the lowest value of $3.7 \times 10^{-14}$ $\mathrm{cm}^{2} / \mathrm{s}[31]$ and the highest value of $2 \times 10^{-11}$ [32]. The diffusivity maximum is recognized clearly from $1600 \mathrm{~K}$ up to $2400 \mathrm{~K}$ under a pressure of $20 \mathrm{GPa}$. The location of the maximum suggested by the previous studies is at $30-40 \mathrm{GPa}$ at $6000 \mathrm{~K} \mathrm{[4]} \mathrm{or} 20 \mathrm{GPa}$ from $2100 \mathrm{~K}$ up to $2750 \mathrm{~K}$ [6]. When comparing the present with the previous study [6], a common feature is that the diffusivity maximum above the glass transition temperature gets weaker and weaker and finally disappears with the increase of temperature. On the other hand, a new finding in this study is that the diffusivity at ambient pressure increases upon compression below $3400 \mathrm{~K}$ (i.e. positive slope in Fig.2); in contrast, the diffusivity decreases above $3600 \mathrm{~K}$ (i.e. negative slope in Fig.2).

To correlate the observation of the diffusivity maximum with structural changes, two profiles of coordination numbers around Si atoms are calculated and shown in Fig.3. Two temperatures, i.e. 2000 $\mathrm{K}$ and $4000 \mathrm{~K}$, are chosen as representative states in which the former has the diffusivity maximum, while in contrast, the latter has not. First, in the case calculated at $2000 \mathrm{~K}$, the ratio of four-fold coordination decreases monotonically upon compression. In line with its decrease, the ratio of five-fold coordination increases. The increase in the ratio of six-fold coordination starts to occur around $20 \mathrm{GPa}$. In contrast, in the case calculated at $4000 \mathrm{~K}$, the ratio of four-fold coordination increases at the beginning and then shows a decrease above $8 \mathrm{GPa}$. This finding has not been discussed previously $[4,6]$. On the other hand, the ratio of five-fold coordination increases monotonically, in the same manner with that at $2000 \mathrm{~K}$. The other interesting finding is the monotonic decrease of three-fold coordination at $4000 \mathrm{~K}$ upon compression. Such a decrease is not observed in the case at $2000 \mathrm{~K}$, because there are few three-fold $\mathrm{Si}$ atoms from the beginning of compression. The increase of six-fold coordination at $4000 \mathrm{~K}$ is smaller than that at $2000 \mathrm{~K}$.

\subsection{LOPN analysis}

To investigate local packing states of silica, LOPN results calculated at $200 \mathrm{~K}, 2000 \mathrm{~K}$ and $4000 \mathrm{~K}$ are shown in Fig.4. The case at $200 \mathrm{~K}$ was added for the LOPN analysis so as to compare the liquid states (at $2000 \mathrm{~K}$ or $4000 \mathrm{~K}$ ) with the solids (at $200 \mathrm{~K}$ ) in which the vibrational effects are diminished. First, in the case at $200 \mathrm{~K}$, the most abundant structural fragment is that with the LOPN of 6 , indicating they are similar to both cristobalite and quartz. In this study, this local packing state is named "low-density glass". When pressure is increased up to $10 \mathrm{GPa}$, the location of the LOPN peak moves from 6 to 8 , showing that its packing state is close to that of coesite, which is a crystal with 
high-density four-fold $\mathrm{Si}$ coordination. In this study, the latter local packing state is named "high-density glass". With further compression from $20 \mathrm{GPa}$ up to $30 \mathrm{GPa}$, the peak shifts to 11 gradually and its packing state is close to that of stishovite that has the LOPN of 12 accompanied by five-fold or six-fold coordination. Next, in the case at $2000 \mathrm{~K}$, the shape and change of LOPN distribution upon compression is almost same as that at $200 \mathrm{~K}$. However, two interesting differences are recognized between $200 \mathrm{~K}$ and $2000 \mathrm{~K}$. The first is the peak height with the LOPN of 6 under 0 GPa decreases at $2000 \mathrm{~K}$, indicating the LOPN distribution becomes broader. The second is the peak under both $20 \mathrm{GPa}$ and $30 \mathrm{GPa}$ shifts down from 10 to 9 and from 11 to 10 at $2000 \mathrm{~K}$, indicating packing is more loosened. Finally, in the case at $4000 \mathrm{~K}$, the distribution at either pressure becomes further broadened compared with either at $200 \mathrm{~K}$ or $2000 \mathrm{~K}$, indicating the structure is more disordered. In addition, the peak shifts downward at either pressure, suggesting the packing is more loosened at the higher temperature. 


\section{Discussion}

The mixed effects originating from the thermally-induced and the pressure-induced complicate the structural transformations occurring in liquid silica. Before investigating liquid silica at high temperature under high pressure, two effects are discussed separately.

\subsection{Thermal effects only}

When silica glass is heated up at ambient pressure, different types of structural transformations occur in sequence. First, the key transformations are briefly reviewed in terms of the 'structon' analysis with which the transformations are explained by four structons, e.g. $\alpha-, \beta-, \gamma$ - and $\delta$-structons (Fig.5). The analysis is applied only for units with 4-fold coordinated Si. If simplified, $\alpha$ - and $\beta$ structons represents a bonded $\mathrm{SiO}_{4}$ dimer $\left(\mathrm{Si}_{2} \mathrm{O}_{7}\right)$ without and with active torsional movements in the solid state. In contrast, $\gamma$ - and $\delta$ - structons represents a $\mathrm{SiO}_{4}$ dimer frequently accompanied by $\mathrm{Si}-\mathrm{O}$ bond breaking and with non-bonded $\mathrm{O}$ (i.e. corresponding to 3-fold coordinated $\mathrm{Si}$ atoms) in the liquid state. Up to the glass transition temperature, the conversion from $\alpha$-structon to $\beta$-struncton (from Fig.1(a) to Fig.1(b)) occurs gradually, showing that the active torsional movement between the $\mathrm{SiO}_{4}$ dimer dominates. This transformation invokes the thermo-mechanical anomaly [13]. Above the glass transition temperature, the $\beta$-structon population saturates and then starts converting into the $\gamma$-structon gradually due to the Si-O bond breakage (from Fig.1(b) to Fig.1(c)), indicating that the expanded structure starts breaking down leading to the density maximum. The presence of $\gamma$-structon implies that even if a bond is broken, it will be re-bonded in a short time keeping four-fold coordination around $\mathrm{Si}$ atoms. This microscopic mechanism is speculated to be the reason why liquid silica is viscous with a weak sign experimentally of the glass transition compared with the other silicate liquids. Above 2000-2500K, $\gamma$-structons gradually convert into $\delta$-structons (from Fig.1(c) to Fig.1(d)). The existence of the $\delta$-structon is equivalent to the existence of four-fold coordination silicon atoms with non-bridging oxygen. In addition, substantial amount of 3-fold coordination (a fraction of $\sim 0.45$ at ambient pressure) at $4000 \mathrm{~K}$ has already been shown in Fig.3. It is interesting to note that neither the $\gamma$ - or $\delta$-structon exists in so-called 'normal liquids' like alkali-rich silicate liquids, in contrast to so-called 'anomalous liquids' like $\mathrm{SiO}_{2}$. We speculate that the existence of the $\gamma$ - or $\delta$-structon invokes the thermo-mechanical anomaly and the density maximum [12].

Several MD studies suggest that there exists a fragile-to-strong transition, e.g. a transition from a fragile liquid at high temperature to a strong liquid at low temperature [e.g.7]. The hypothesis mainly comes from the inflection in the T dependence energy or heat capacity in the previous study [7]. The estimated temperature by using the BKS potential was around $4000 \mathrm{~K}$ under constant volume calculation [7]. On the other hand, no experimental data are available. In contrast, in terms of structural transformation in liquid states, the structon analysis in this study suggests there occurs a gradual conversion from $\gamma$-structon at low temperature to $\delta$-structons at high temperature. When the 
temperature increases just above the glass transition temperature where the $\gamma$-structon dominates the whole structure (the zone between $1500 \mathrm{~K}$ and $3200 \mathrm{~K}$ in Fig.5), the number of Si-O bonds is almost constant (i.e. almost four-coordination around Si atoms). Only the frequency of bond-breaking and bond-rebonding events changes due to an increase in the kinetic energy, while keeping the same potential energy barrier. Therefore, we speculate that the Arrhenius formula itself for thermal activation does not change. In contrast, when the temperature increases in the high temperature range where the $\delta$-structons increase steadily (the zone above $3600 \mathrm{~K}$ in Fig.5), the number of non-bonded $\mathrm{O}$ atoms and three-fold coordinated Si atoms increases. In the other words, the potential energy barrier changes as well as kinetic energy at higher temperatures. If the origin of fragile properties in modifier-rich silicate glass comes from the bond-breaking between non-bonded $\mathrm{O}$ and modifiers atoms, we speculate that the increase of non-bonded $\mathrm{O}$ atoms and three-fold coordinated $\mathrm{Si}$ atoms enhance fragile properties, e.g. the average activation energy estimated by using Arrhenius formula would not be same, but depend on temperature. Considering that the boiling point of silica is $2505 \mathrm{~K}$ and the soft potential could reproduce the melting structures better than the BKS potential, the emergence of 3-fold $\mathrm{Si}$ atoms might be a trigger to vaporization. Therefore, we speculate that it would be difficult to observe the fragile-to-strong transition at ambient pressure experimentally.

In association with this structural change, the other clue is provided by the pressure dependence of diffusivity at ambient pressure shown in Fig.2. This dependence is positive and negative depending on the temperature, i.e. below or above $3400 \mathrm{~K}$. As shown in Fig.3, almost all the $\mathrm{Si}$ is in four-coordination at $2000 \mathrm{~K}$ and ambient pressure. The number of four-fold Si decreases gradually being replaced with five-fold upon compression. Therefore, a well-developed Si-O network is maintained just above the glass transition temperature even if the distortion of local structure enhances the diffusivity. On the other hand, only half of the Si atoms have four-fold coordination at $4000 \mathrm{~K}$. Upon compression, three-fold coordination is converted into four-fold. In other words, the change of three-fold coordination is sensitive to pressure and thermal changes.

To summarise these results, the broad temperature region whose centre is around $3200-3400 \mathrm{~K}$ is considered to be the zone of the fragile-to-strong transition, regarding which the abrupt increase of four-fold coordination with non-bridging oxygen (i.e. the birth of $\delta$-structons) starts around $2500 \mathrm{~K}$ and then the pressure dependence of diffusivity turns to almost zero around 3200-3400 K (i.e. the saturation of $\gamma$-structon).

\section{2 pressure effects only}

As for the pressure effects at ambient temperature, three main phenomena have been discussed [e.g.26]. The first is the elastic modulus anomaly: the elastic modulus decreases upon compression and has its minimum value at $2 \mathrm{GPa}$. The anomaly is reversible and its origin is explained by the several rotations of Si-O-Si planes, in common with the $\alpha$-to- $\beta$ cristobalite transition, where the elastic 
modulus anomaly occurs around $7 \mathrm{GPa}$ [27]. It is interesting to note that the five-fold coordination starts increasing over $5 \mathrm{GPa}$ in the author's previous study [22]. This pressure is suspected to be the onset of the elastic anomaly, because the glass can be compressed into the compact structure still preserving four-fold coordination up to $5 \mathrm{GPa}$; however, the coordination has to increase to five-fold above this pressure. This region corresponds with low-density glass in terms of the LOPN.

The second phenomenon is the permanent densification. The density increases after decompression and the permanent densification occurs about 5-10 GPa. The densification saturates around $20 \mathrm{GPa}$. Not only MD simulation [e.g.22,26,28] but also experimental studies [e.g.1,29] have discussed its microscopic origin. After bond-breaking upon compression, rebonding occurs and new compact arrangements of Si-O are generated. The present author showed that five-fold coordination increases monotonically between 5 and $20 \mathrm{GPa}$ [33]. Therefore, five-fold coordination would be the transient route which allows the bond-breaking and the rebonding to take place. Finally, it is now widely recognized that there are two forms of silica glass, e.g. low-density and high-density glass, each with four-fold coordination around $\mathrm{Si}$ atoms. As discussed in the Results section, the packing patterns can be distinguished between these two types of glass in terms of the LOPN. In other words, the denser glass has the greater fraction of coesite-like packing.

The third phenomenon is that structures with a higher-coordination number appear over around $20 \mathrm{GPa}$ during compression. It was demonstrated that $\alpha$-cristobalite transforms into hp-cristobalite and finally into six-fold coordinated Stishovite, explaining that such two-stage densification is a ubiquitous mechanism [29]. The present author also showed that proportion of six-fold coordinated $\mathrm{Si}$ atoms starts increasing over 20 GPa monotonically; however, such coordination is not quenchable [22]. In this region, the ultra-high density structure becomes more and more dominant in terms of the LOPN.

\subsection{Mixed effects of high temperature and high pressure}

The mixed effects are discussed using two approaches. The first is based on the liquid structure at $2000 \mathrm{~K}$, which is compressed from ambient up to $30 \mathrm{GPa}$ (Fig. 7(a)). Just above the glass transition temperature, almost all the $\mathrm{Si}$ atoms have four-coordination. As the pressure increases up to around 20 $\mathrm{GPa}$, five-fold coordination changes into four-fold coordination. Energetically activated five-fold environments would help overcoming the energy barrier for diffusion. However, the further compression also increases six-fold coordination that blocks diffusion and finally there is a substantial decrease in diffusion. During the whole process, the low-density liquid changes into the ultra-high liquid with high density.

The second approach considers the liquid structure at $4000 \mathrm{~K}$, which is compressed from ambient pressure up to $30 \mathrm{GPa}$ (Fig.7(b)). At ambient pressure, there is abundant three-fold coordination. As the pressure increases, three-fold changes into four-fold rather than five-fold 
coordination. . The decrease of three-fold coordination would raise the energy barrier and decrease diffusion. When the pressure is increased up to $20 \mathrm{GPa}$, mixed effects, i.e. decrease of three-fold and increase of five-fold coordination occur. Some are cancelled out and the total effect leads to the weak or zero decrease of diffusion. Even under $30 \mathrm{GPa}$, diffusion shows a weak decrease; however, in this region the mixed effect, i.e. increase of five-fold and six-fold coordination, dominates diffusion. During the whole process, the low-density liquid changes into the ultra-high liquid with high density in a similar manner to the case at $2000 \mathrm{~K}$; however, the thermal effects slightly decelerate the densification.

The remaining problem is the explanation of why the diffusivity increases from 0 to $5 \mathrm{GPa}$ below $2000 \mathrm{~K}$ although the structure keeps 4-fold coordination. In the discussions so far, two mechanisms enhance the diffusivity: one is the creation of non-bonded oxygen atoms accompanied by three-fold coordinated Si atoms at high temperature and ambient pressure; the other is the creation of five-fold coordinated $\mathrm{Si}$ at high pressure. At pressures from 0 to $5 \mathrm{GPa}$, the snapshot of the structure shows only the presence of four-coordinated $\mathrm{Si}$ atoms; however, twe speculate that the diffusion occurs via an instantaneous five-fold coordinated $\mathrm{Si}$ environment due to compaction. This speculation might correspond with the hypothesis in which the mixing entropy of bridging and non-bridging oxygen increased upon compression [15]. Further study is required to explain this phenomenon in more detail.

\subsection{Relation with density change}

The calculated densities at two representative temperatures corresponding with Figs. 2, 3 and 4 are shown in Fig.6. The calculated density at $200 \mathrm{~K}$ at ambient pressure is $2.23 \mathrm{~g} / \mathrm{cm}^{3}$, which is close to the experimental value at room temperature of $2.20 \mathrm{~g} / \mathrm{cm}^{3}$. To the authors knowledge, no in-situ experimental value is available either at high temperature or pressure.

Overall, as the pressure increases, the density increases monotonically. In other words, the increase in coordination number either around $\mathrm{Si}$ or $\mathrm{O}$ leads to the increase in density. There is no distinct change in density around $20 \mathrm{GPa}$ in which the diffusivity maximum is observed, which therefore suggests the necessity of detailed local analysis such as coordination numbers and packing states shown in this study.

The remaining problem is to compare the free energies between three-, four-, five-, and six-fold environments to amplify our understanding of the above-mentioned scenario, which will be the topic of further studies. 


\section{Conclusions}

In this study, structural changes of liquid silica were investigated under high pressure by molecular dynamics simulation. The 'soft potential' model was employed, because the real phenomena are expected to be reproduced at the appropriate temperatures. The diffusivity and the coordination number around $\mathrm{Si}$ atoms were calculated and analyzed. Next, to advance further analyses, the LOPN method that the author developed in the previous studies, was employed. First, it was demonstrated that the diffusivity maximum near the glass transition temperature corresponded with the structural change from four-, to five-, and six- fold coordinated Si environments, in particular, the emergence of five-fold and six-fold coordination as speculated in the previous studies $[3,6]$. In addition, several new findings were explained on the basis of complex structural changes. It has been overlooked previously that the conversion between three-fold and four-fold coordination is an important factor. Not only does the diffusivity maximum disappear above $3400 \mathrm{~K}$, with the liquid silica then behaving like a normal liquid, but also the pressure dependence of diffusivity at ambient pressure changes its sign around $3400 \mathrm{~K}$. At $2000 \mathrm{~K}$, just above the glass transition temperature, compression creates the five-fold coordination and enhances the diffusivity at a pressure from 5 to 20 GPa. In contrast to low temperature, compression converts three-fold into four-fold coordination leading to the decrease in diffusivity at high temperatures (e.g. $4000 \mathrm{~K}$ ).

In summary, two mechanisms enhance the diffusivity in silica liquids. One is the creation of non-bonded oxygen accompanied by three-fold coordinated $\mathrm{Si}$ atoms at high temperature and ambient pressure. The other is the creation of five-fold coordinated Si at high pressure.

The structural analysis shed new light on the fragile-to-strong transition. In the case of anomalous liquids, the author represented the structural transformation with only four simplified structons, ie., $\alpha-, \beta-, \gamma$ - and $\delta$-structons. As discussed, the existence of $\beta$ - and $\gamma$-structons invokes several anomalies. If the $\gamma$-rich structure preserves the Si-O network even if the breaking and rebonding are repeated frequently, the liquid would behave like strong liquid. In contrast, when several non-bonding sub-structures represented as $\delta$-structons increase upon heating, the system would behave like fragile liquid. 


\section{References}

[1] R. Brückner, J. Non-Cryst. Solids 5 (1970) 123.

[2] H.T. Smyth, J. Am. Ceramic. Soc. 38 (1955) 140.

[3] L.V. Woodcock, C.A. Angell, P. Cheeseman, J. Chem. Phys. 65 (1976) 1565.

[4] C.A. Angell, P.A. Cheeseman, S. Tamaddon, Science 218 (1982) 885.

[5] S. Tsuneyuki, Y. Matsui, Phys. Rev. Lett. 74 (1995) 3197.

[6] J. Horbach, J. Phys.: Condens. Matter 20 (2008) 244118.

[7] I. Saika-Voivod, P.H. Poole, F. Sciortino, Nature 412 (2001) 514.

[8] K. Vollmayr, W. Kob, K. Binder, Phys. Rev. B 54 (1996) 15808.

[9] J. Horbach, W. Kob, Phy. Rev. B 60 (1999) 3169.

[10] K. Yamahara, K. Okazaki, K. Kawamura, J. Non-Cryst. Solids 291 (2001) 32.

[11] M.S. Shell, P.G. Debenedetti,A.Z. Panagiotopoulos, Phy. Rev. E 66 (2002) 011202.

[12] A. Takada, P. Richet, C.R.A. Catlow, G.D. Price, J. Non-Cryst. Solids $345 \& 346$ (2004) 224.

[13] L. Huang, L. Duffrène, J. Kieffer, J. Non-Cryst. Solids 349 (2004) 1.

[14] G.H. Wolf, P.F. McMillan, in: J.F. Stebbins, P.F. McMillan, D.B. Dingwell (Eds.), Structure, Dynamics and Properties of Silicates Melts (Reviews of Mineralogy Vol.32), Mineralogical Society of America, Washington,1995, p.505.

[15] P. Richet, Y. Bottinga, in: J.F. Stebbins, P.F. McMillan, D.B. Dingwell (Eds.), Structure, Dynamics and Properties of Silicates Melts (Reviews of Mineralogy Vol.32), Mineralogical Society of America, Washington, 1995, p.67.

[16] B.W.H. van Beest, G.J. Kramer, R.A. van Santen, Phys. Rev. let. 64 (1990) 1955.

[17] E. Lascaris, M. Hemmati, S.V. Buldyrev, H.E. Stanley, C.A. Angell, J. Chem. Phys. 140 (2014) 224502.

[18] E. Lascaris, M. Hemmati, S.V. Buldyrev, H.E. Stanley, C.A. Angell, J. Chem. Phys. 142 (2015) 104506.

[19] C.A. Angell, Int.J. Appl. Glass Sci. 6 (2015) 3.

[20] A. Takada, Eur. J. Glass Sci. Technol. B 50 (2009) 219.

[21] B.B. Karki, D. Bhattarai, L. Strude, Phys. Rev. B 76 (2007) 104205.

[22] M. Wilson, P.A. Madden, M. Hemmati, C.A. Angell, Phys. Rev. Lett. 77 (1996) 4023.

[23] A. Takada, P. Richet, C.R.A. Catlow, G.D. Price, Eur. J. Glass Sci. Technol. B 48 (2007) 182.

[24] A. Takada, P. Richet, C.R.A. Catlow, G.D. Price, J. Non-Cryst. Solids 354 (2008) 181.

[25] T.F. Soules, G..H. Gimer, M.J. Matthews, S. Stolken, M.D. Feit, J Non-Cryst Solids 357 (2011) 1564.

[26] L. Stixrude, in: R.A. Devine, J.-P. Duraud, E. Dooryhée (eds.), Structure and Imperfections in Amorphous and Crystalline Silicon Dioxide, Wiley, Chicester. 2000, p.69. 
[27] L. Huang, J. Kieffer, Phys. Rev. B 69 (2004) 224203.

[28] L. Huang, J. Kieffer, Phys. Rev. B 69 (2004) 224204.

[29] B. Champagnon, C. Martinet, M. Boudeulle, D. Vouagner, C. Coussa, T. Deschamps, L. Grosvalet, J. Non-Cryst. Solids 354 (2008) 569.

[30] I. Saika-Voivod, F. Sciortino, T. Grande, P.H. Poole, Phys. Rev. E 70 (2004) 061507.

[31] J.C. Mikkelson, Appl. Phys. Lett. 45 (1984) 1187.

[32] E.W. Sucov. J. Am. Ceram. Soc. 46 (1963) 14.

[33] A. Takada, P. Richet, T. Atake, J. Non-Cryst. Solids 356 (2010) 2486. 


\section{Figure captions}

Fig.1 Tetrahedral representation of four 'structon'; (a) $\alpha$-structon with inactive movement in strong bonding state, (b) $\beta$-structon with active torsional movement in strong bonding state, (c) $\gamma$-structon in flexible bonding state, and (d) $\delta$-structon in non-bonding state. One tetrahedron is comprised of a $\mathrm{SiO} 4$ unit.

Fig.2 Profile of pressure dependence of diffusivity.

The unit of diffusivity is $\left[\mathrm{cm}^{2} / \mathrm{s}\right]$. The dotted circle is a noticeable point at which the diffusivity shows its diffusivity maximum. The dashed circles indicate the area of changing sign of pressure derivative.

Fig.3 Profile of pressure dependence of coordination number around $\mathrm{Si}$.

(a) Calculated results at $2000 \mathrm{~K}$. (b) Calculated results at $4000 \mathrm{~K}$.

Fig.4 Results of LOPN analysis for silica.

Calculated at t $200 \mathrm{~K}(\mathrm{a})$, at $2000 \mathrm{~K}(\mathrm{~b})$, and at $4000 \mathrm{~K}$ (c).

Fig.5 Structon analysis for silica.

The 'structon' analysis is only applied for $\mathrm{Si}_{2} \mathrm{O}_{7}$ units comprised of 4-fold coordinated $\mathrm{Si}$ atoms.

Fig.6 Calculated density profiles.

Fig.7 Speculated scenario on changing mechanism of diffusivity.

(a) At 2000K. (b) At 4000K. 
Fig.1 Tetrahedral representation of four 'structons'; (a) $\alpha$-structon with inactive movement in strong bonding state, (b) $\beta$-structon with active torsional movement in strong bonding state, (c) $\gamma$-structon in flexible bonding state, and (d) $\delta$-structon in non-bonding state. One tetrahedron is comprised of a $\mathrm{SiO} 4$ unit.

(a)

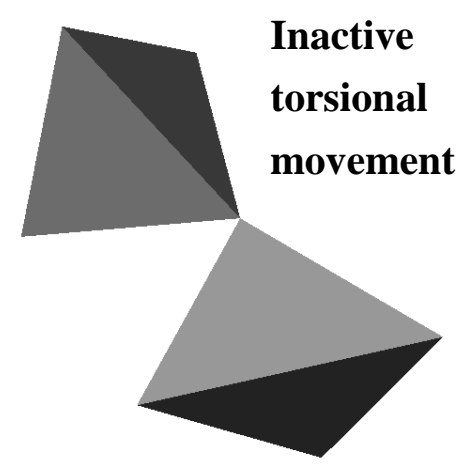

(b)
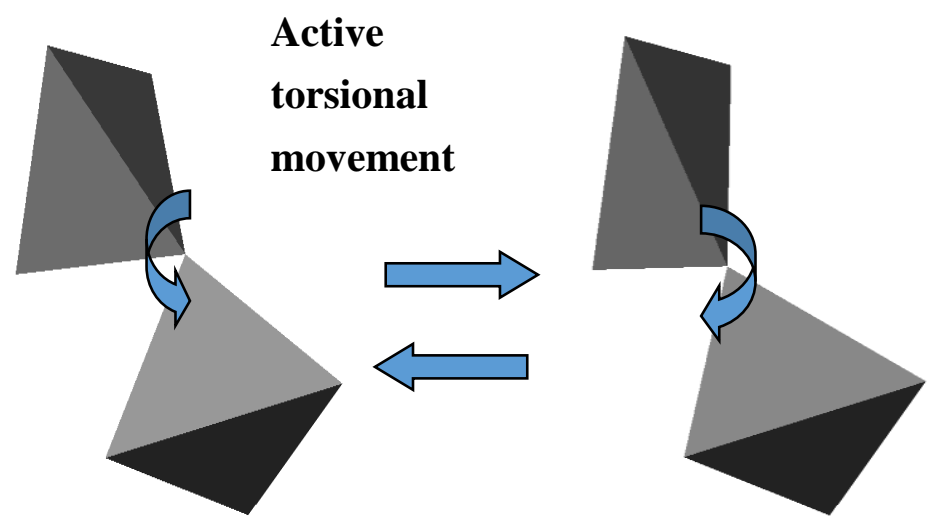
(c)

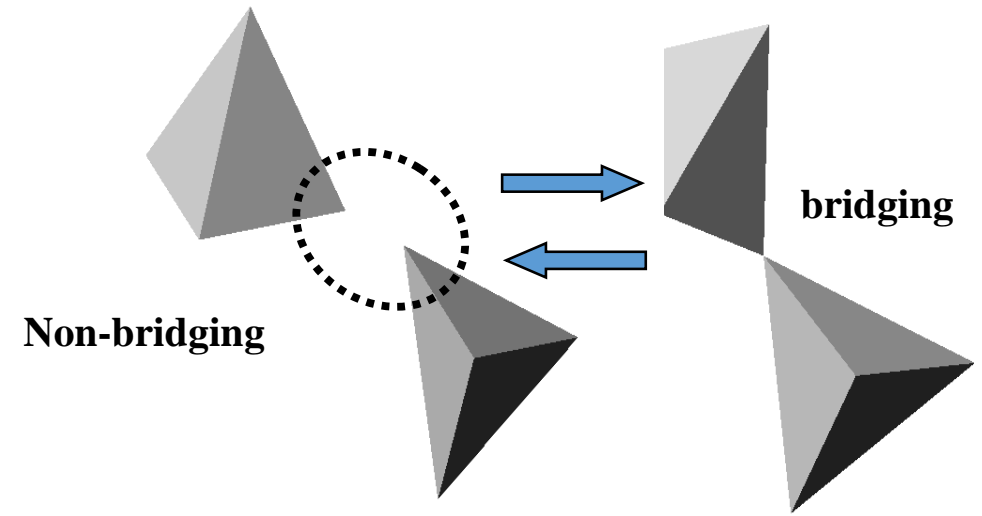

(d)

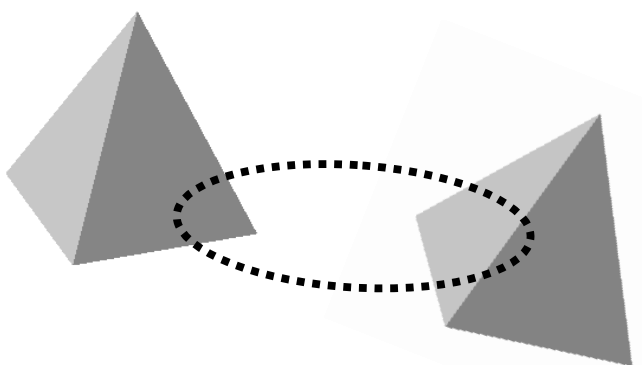

Non-bridging 
Fig.2 Profile of pressure dependence of diffusivity.

The unit of diffusivity is $\left[\mathrm{cm}^{2} / \mathrm{s}\right]$. The dotted circle is a noticeable point at which the diffusivity shows its diffusivity maximum. The dashed circles indicate the area of changing sign of pressure derivative.

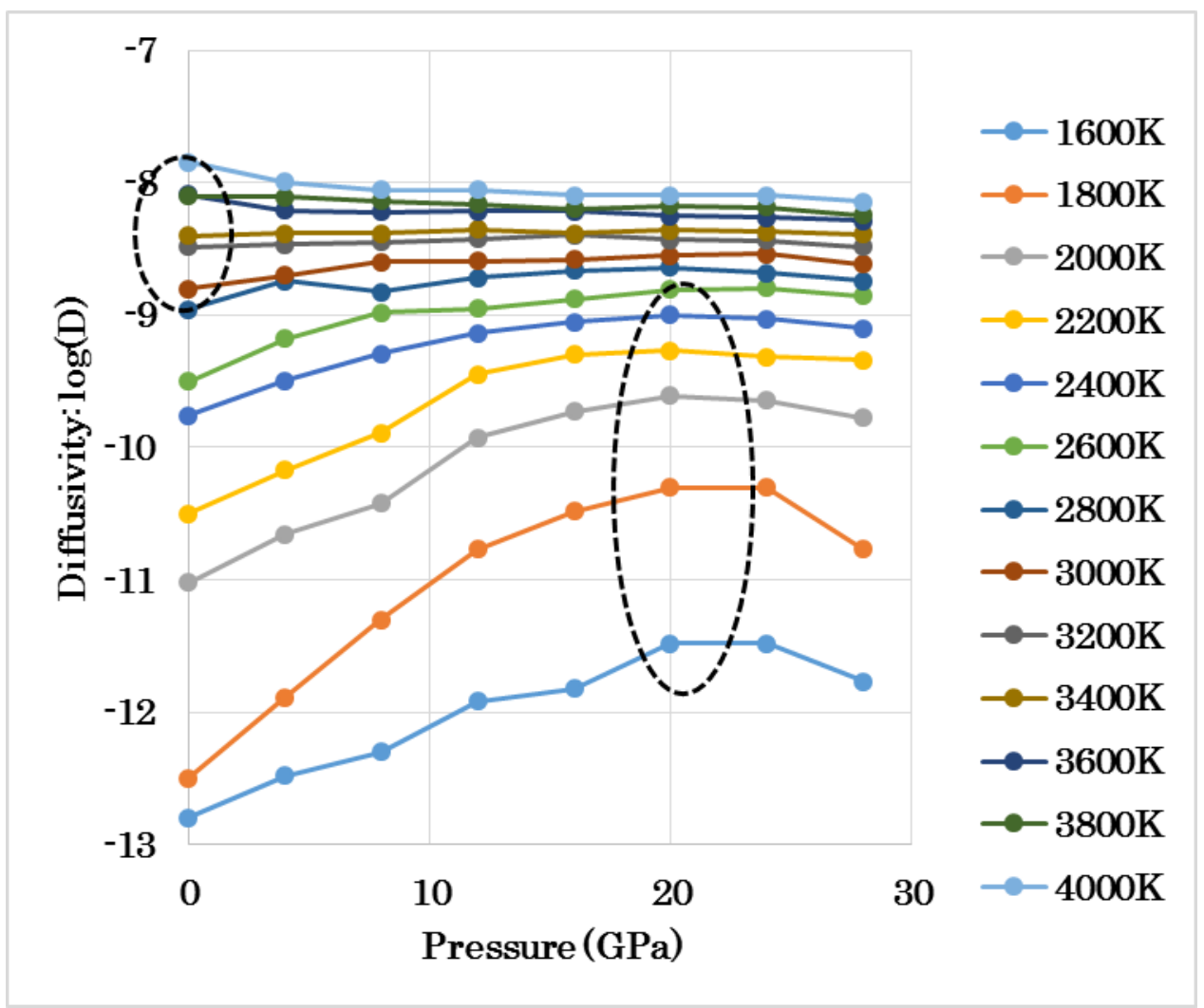


Fig.3 Profile of pressure dependence of coordination number around Si.

(a) Calculated results at $2000 \mathrm{~K}$. (b) Calculated results at $4000 \mathrm{~K}$.

(a) Calculated results at $2000 \mathrm{~K}$.

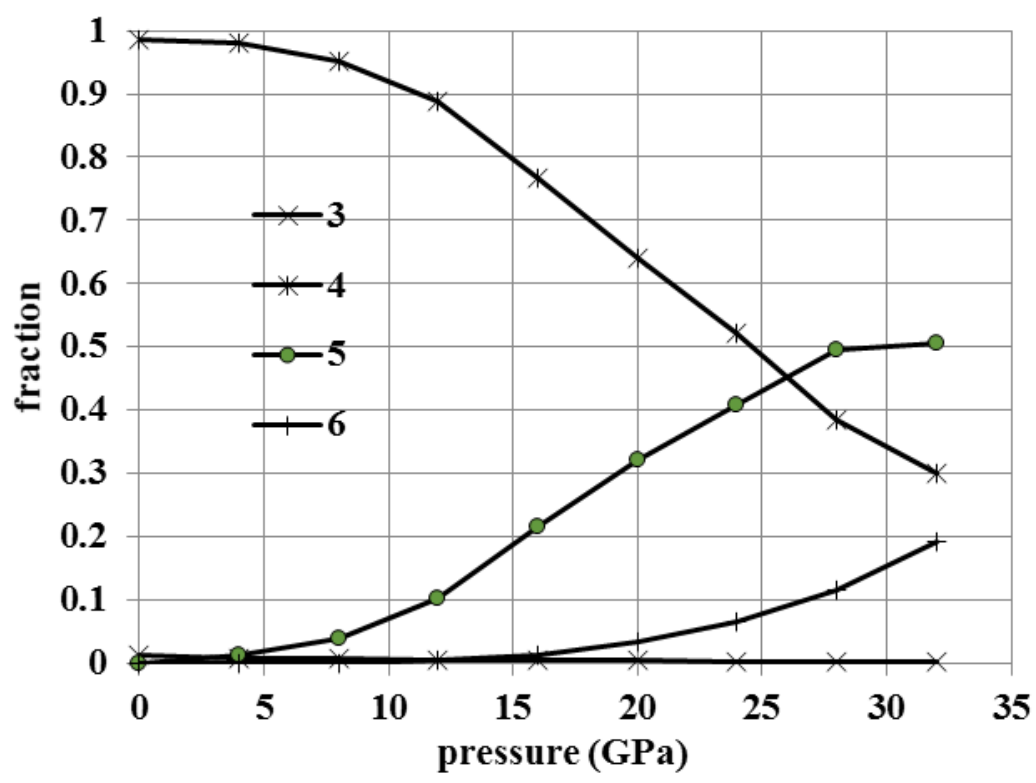

(b) Calculated results at $4000 \mathrm{~K}$.

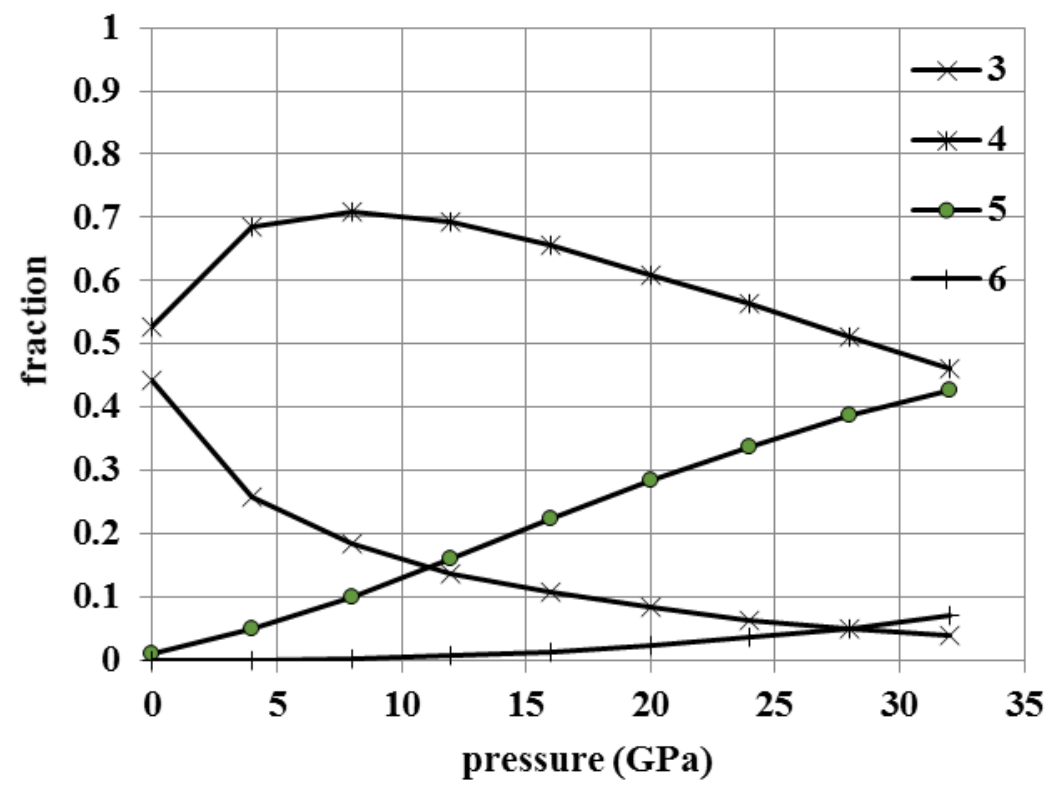


Fig.4 Results of LOPN analysis for silica.

Calculated at t $200 \mathrm{~K}$ (a), at $2000 \mathrm{~K}$ (b), and at $4000 \mathrm{~K}$ (c).

(a) At $200 \mathrm{~K}$.

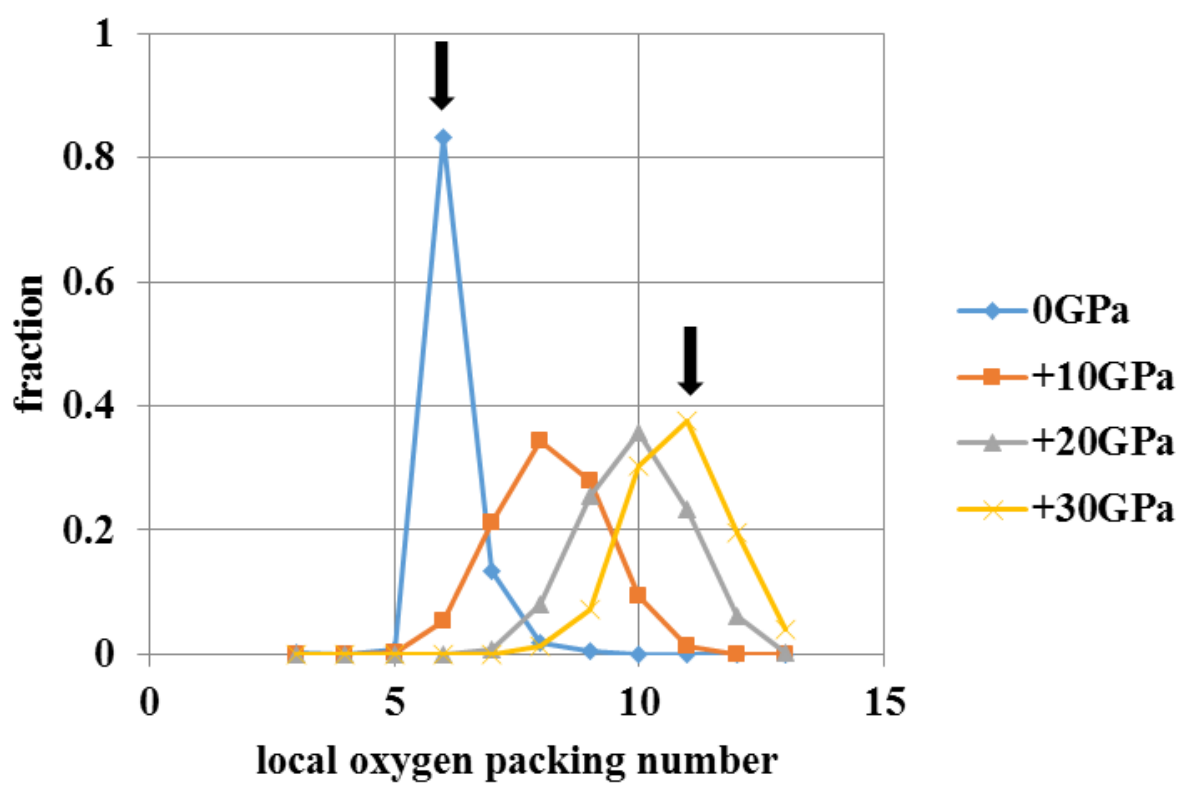

(b) At $2000 \mathrm{~K}$.

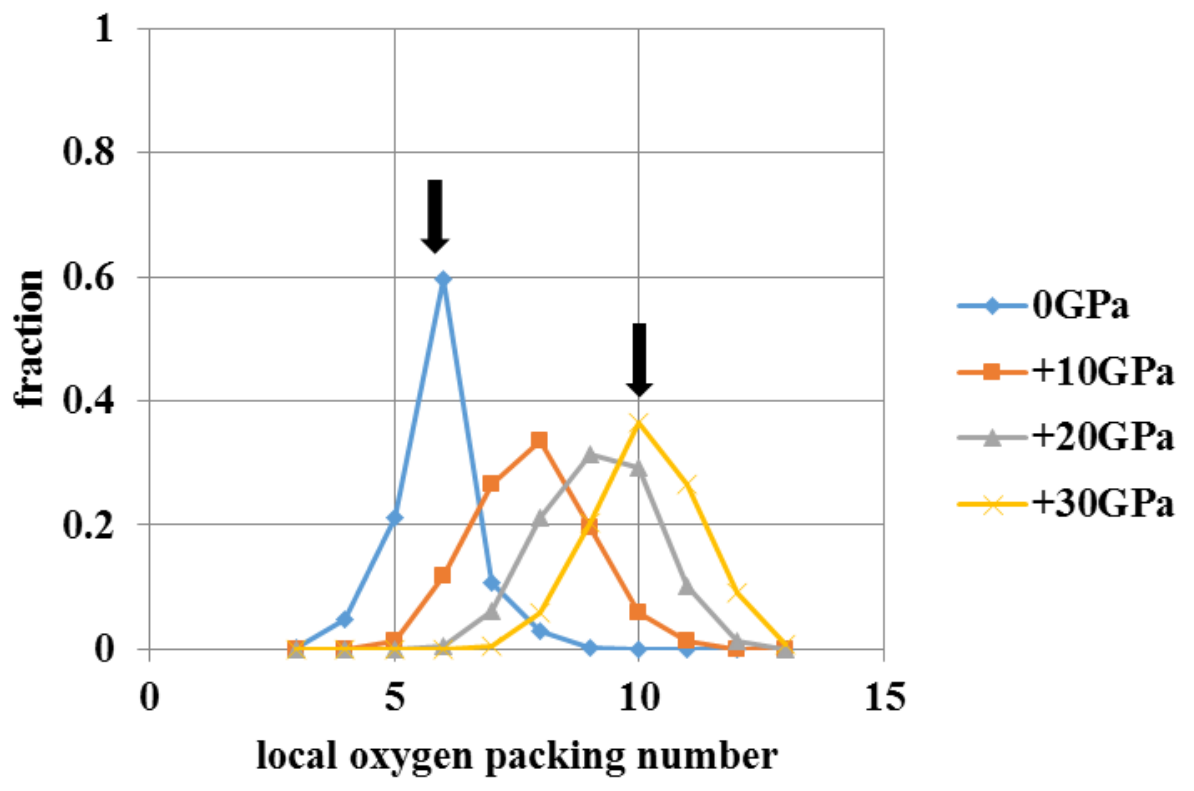


(c) At $4000 \mathrm{~K}$.

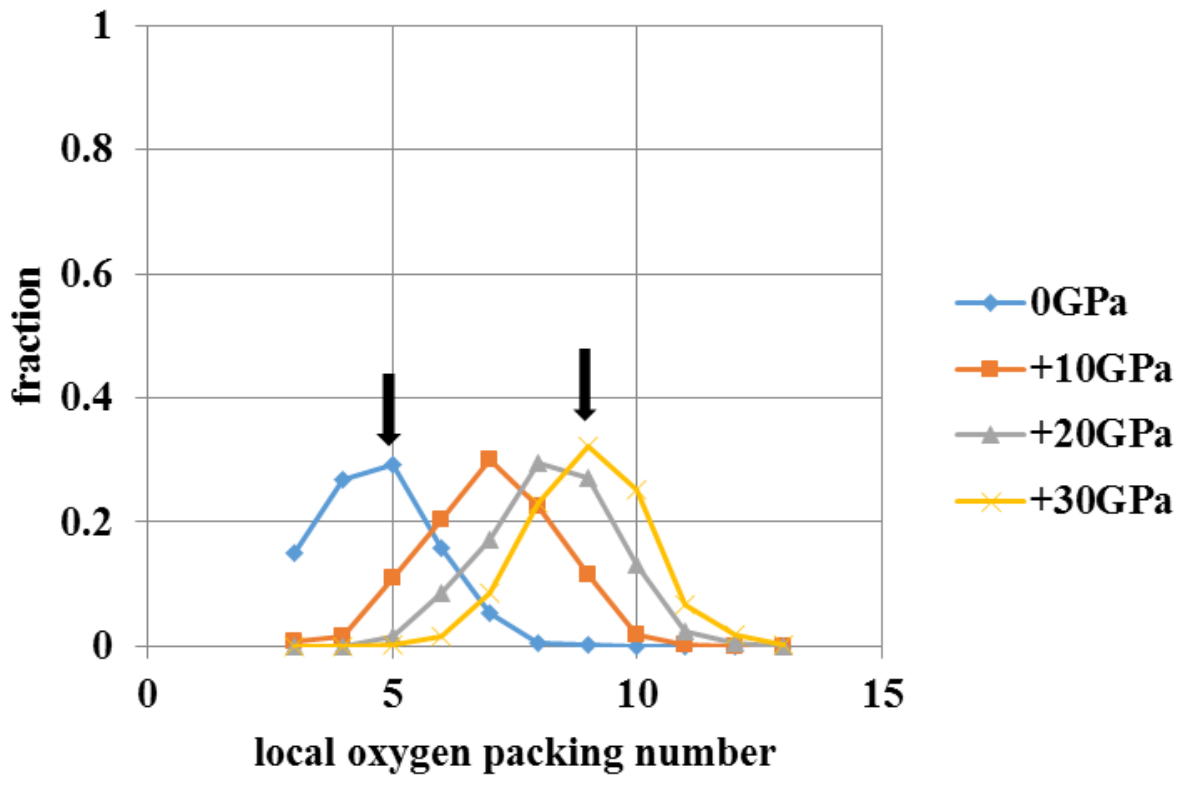


Fig.5 Structon analysis for silica.

The 'structon' analysis is only applied for $\mathrm{Si}_{2} \mathrm{O}_{7}$ units comprised of 4-fold coordinated $\mathrm{Si}$ atoms.

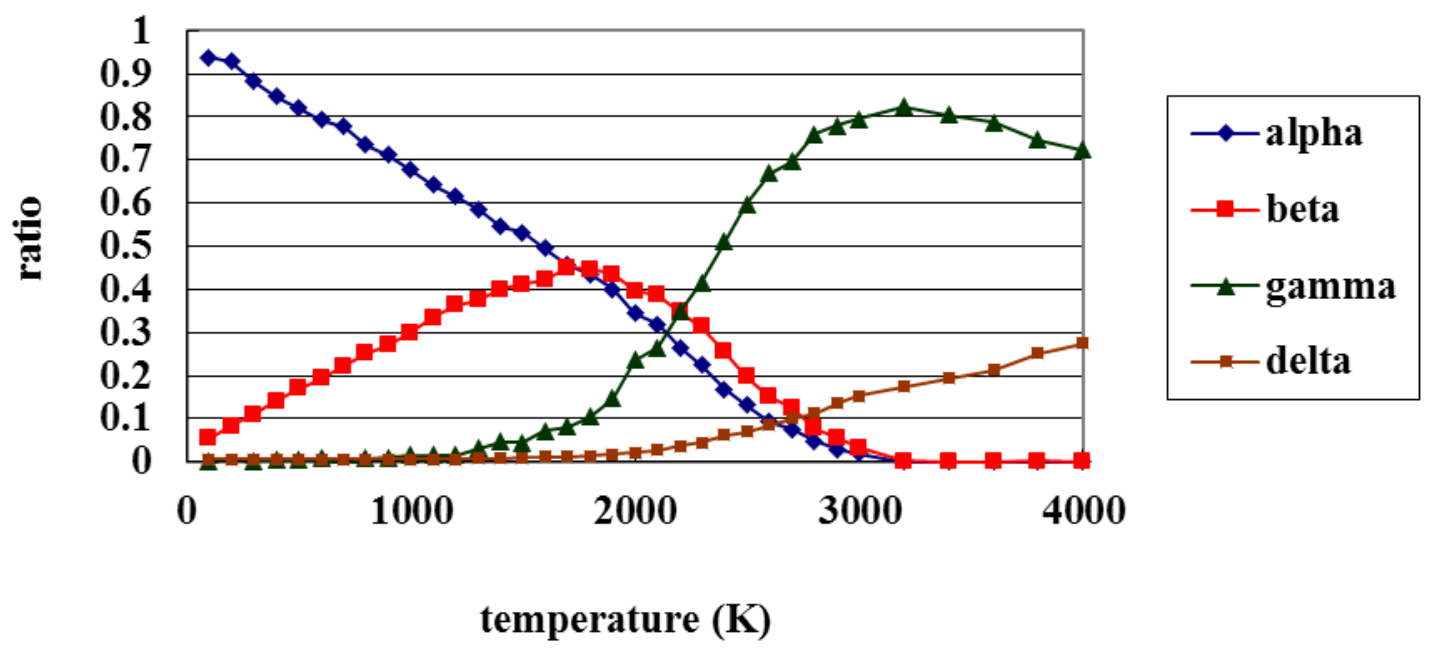


Fig.6 Calculated density profiles.

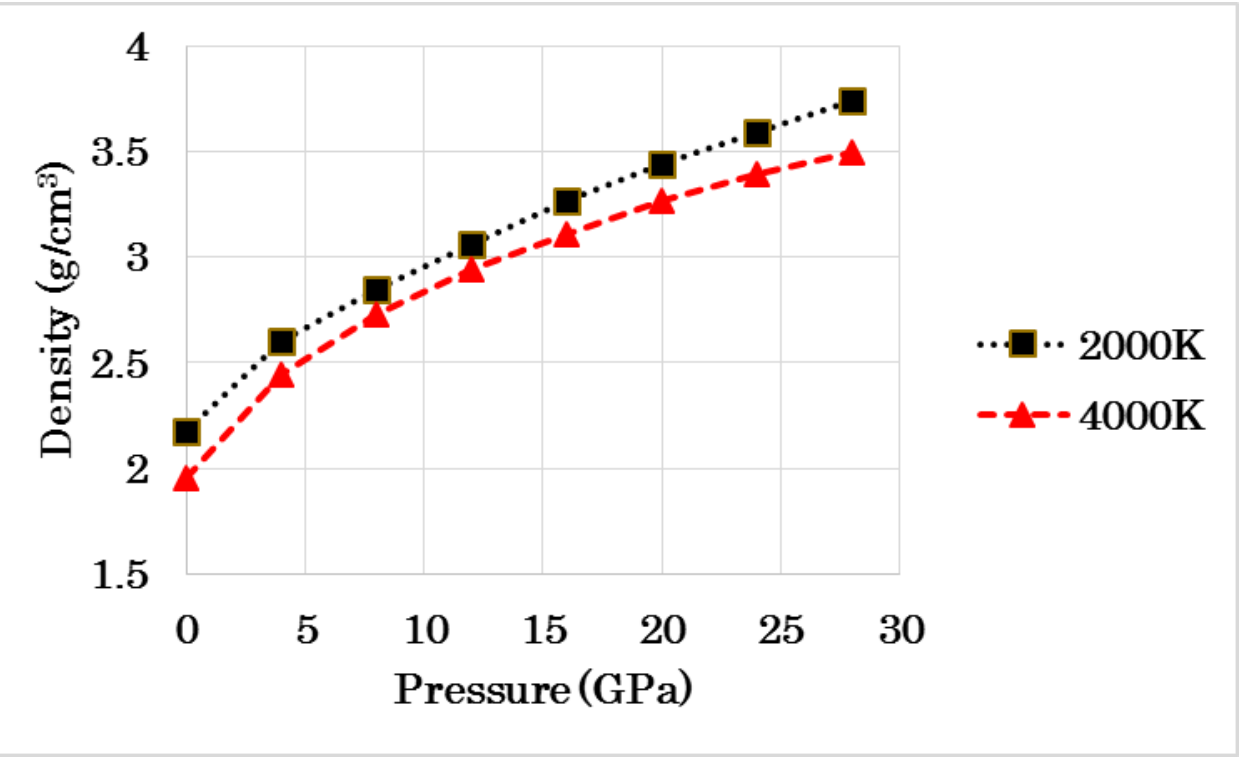


Fig.7 Speculated scenario on changing mechanism of diffusivity.

(a)At $2000 \mathrm{~K}$.

Around $0 \mathrm{GPa}$

energetically stable

configuration

(low-density liquid)

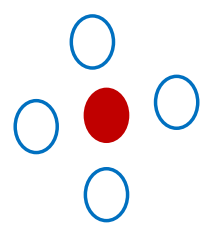

$\underline{\text { Around } 20 \mathrm{GPa}}$

generation of

five-fold Si environments

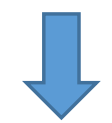

$\rightarrow$ strong increase of D

(mixture of high and

ultra-high density)

\section{Over $30 \mathrm{GPa}$}

generation of

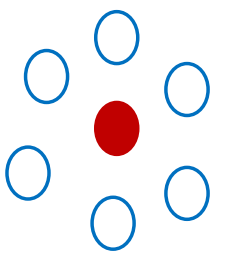

six-fold Si environments

$\rightarrow$ strong decrease of D

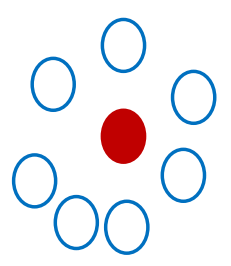

(mainly ultra-high density) 
(b) At $4000 \mathrm{~K}$.

\section{Around 0 GPa}

many 3-fold Si environments

$\circ \bullet$

$\rightarrow$ strong increase of $D$

(low-density liquid)

\section{Around $20 \mathrm{GPa}$}

Mixed effects of decrease of three-fold and increase of five-fold

Si environments

$\rightarrow$ weak decrease of D

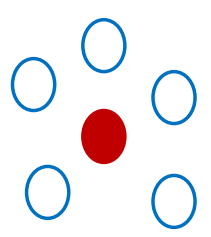

(mainly high-density)

\section{Over $30 \mathrm{GPa}$}

Mixed effects of increase of five-fold and increase of six-fold

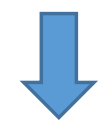

$\mathrm{Si}$ environments

$\rightarrow$ weak decrease of D

(mixture of high and ultra-high density) 